\title{
A NEW METHOD
}

\author{
OF \\ OPERATING FOR THE RADICAL CURE \\ OF HERNIA. \\ BY \\ JOHN WOOD, F.R.C.S.,
}

ASSISTANT-SURGEON TO KING'S COLLEGE HOSPITAL, DEMONSTRATOR OF ANATONY AT KING'S COLLEGE, AND SURGEON TO THE

IINCOIN'S INN DISPENSARY.

\section{Receired Dec. 24th, 1859.-Read Fel. 28th, 1800.}

The operations that have been hitherto devised for the cure of hernia may be classed under three heads, viz.1st. Those which deal with the interior of the sac only, with a view of causing adhesion of its surfaces, either by a seton passed through it, as in Ragg's method, or by the injection of a solution of iodine or some irritating fluid, as practised in America and elsewhere. Without considering the frequent occurrence of peritonitis after the latter plan has been adopted, both are open to the objection that, even supposing that they are effectual in causing adhesion of the sides of the sac, the abdominal openings and the inguinal canal are left as patulous as before; while the loose connexion of the peritoneum with the fascia transversalis; in this situation, permits it readily to furnish another sac for a fresh protrusion. 2d. That of pinching up the anterior wall of the inguinal protrusion by a needle passed 
across it through the skin, with the object of causing adhesion of the opposed serous surfaces, as proposed by Bonnet, of Lyons. This method evidently deals only with that part of the hernia which protrudes through the external abdominal ring, leaving the upper part of the sac in the inguinal canal untouched and with a constant tendency to push down. 3d. The method of invagination of the skin into the canal. Upon this principle are founded two operations, that proposed by Gerdy, and the later one practised by Wurzer and Rothemunde.

In Gerdy's method, the skin and fascia covering the sac are invaginated upon the finger of the operator, and held in that position by a ligature thrust through the intercolumnar fascia and skin of the groin, till adhesion takes place at the point of ligature. An endeavour is also made to produce adhesion of the interior surface of the invaginated skin by the use of caustics and ligatures at the lower opening of the invagination; but this, I believe, was a failure. This method evidently deals only with the anterior wall of the hernia, and with the anterior fold of reduplication of the hernial sac. To make this intelligible, I must refer to Pl. IV, fig. 1, in which it will be seen that the reduplication of the sac leaves a posterior fold $(s)$, which, on account of the closer attachment of the sac to the cord in that part, is usually longer, and descends lower than the anterior. The ligature evidently can only produce adhesion of the anterior fold, and other anterior structures, at $a$.

The method proposed by Wurzer substitutes for the finger of the operator a wooden plug, variously modified with the intent to fill the canal and openings, and to stretch them so much as to set up adhesive inflammation all round the invaginated sac. This plug is held in its position by one or two needles passing through the anterior wall, and connected with an external compress, intended to squeeze the anterior fold between it and the plug, so as to produce adhesion of the serous surfaces in its whole length. The essential difference between this method and Gerdy's consists in the retention of the plug till it has set up adhe- 
sive inflammation by its dilating pressure upon the opposed surfaces of the sac and the boundaries of the canal; the theory being that this will take place to such an extent as to prevent the unfolding of the invagination, which is thus supposed to plug up the openings by an inverted hollow cone of skin and fascia, as the neck of a bottle by the cork. The attempt is also sometimes made to cause the sides of the inverted cone to adhere by caustics and pressure.

Now, since in all these methods of producing a radical cure, the sac of the hernia is intended to be punctured and caused to inflame sufficiently to produce adhesion, the danger of peritonitis, which is regarded by many as a serious objection to any operation of this kind, may be considered as pretty nearly equal in all. From this, however, we must except the practice of injecting irritating fluids, which are obviously very likely to pass into the peritoneal cavity, and have often in this way proved fatal. But the results of the numerous cases operated on by the other methods that have been recorded, especially by Wurzer's, seem to prove that the danger of a fatal result is by no means great, nor sufficient to deter the surgeon from endeavouring to perfect our means of cure of this common deformity. In the method I have followed it will be seen, that it is by no means essential in recent and small cases of hernia to puncture the sac, since the most important manipulations can be effected altogether external to it, the sac being separated from its slight attachments and pushed up before the finger; and this, when it can be done, very much diminishes even the small chance there remains of peritoneal inflammation. A much more awkward objection is drawn from the inefficacy of these methods. Without wishing in any way to disparage the results of the operations I have thus briefly alluded to, or to magnify my own plan at the expense of others, I may say that in the hands of those surgeons from whom $I$ have been able to obtain an account of the results of Wurzer's (the last and most extensively employed) and in all those cases which have come under my own observation, it 
has been entirely unsatisfactory; the rupture re-descending either immediately on leaving off the instrument and assum. ing the erect posture, or after discontinuing the truss worn after the operation. And this, I believe, is a very general impression among the surgeons in this country, as well as in France and Germany. In some cases I have heard of, it has rendered the wearing of a truss afterwards painful, if not impossible.

The causes of failure appear to me to be the following:

1st. The chief reason of the recurrence of the hernia, both in Gerdy's and Wurzer's operations, is the inefficiency of the steps taken to cause adhesion of the surfaces of the posterior fold of the invaginated sac together, and to the posterior wall of the hernial canal. Into this fold, forming thus a secondary sac, the descent of a knuckle of intestine or omentum is imminent, and its dilatation, and the consequent unfolding of the adherent portion in front, is only a question of time. In Gerdy's method no attempt is made for the closure of the posterior fold. In Wurzer's, the dilating pressure of the plug is supposed to produce this adhesion. To this theory the yielding nature of the structures, and the impossibility of producing a necessary amount of dilatation and pressure, without unbearable pain, offer the first objection.

2d. The intervention of the skin and fascia between the plug, the sac, and the parietes of the canal in which it is intended to produce adhesion. These are structures intended by nature to protect the subjacent parts invested by them from the effects of pressure and injury. Here we have the exertions of nature against those of the surgeon, and generally, I believe nature to have the best of it. It is said, moreover, that those cases make the most permanent cures in which sloughing of the skin and fascia at the apex of the plug takes place. This result, though effectual in overcoming the resistance of the tissues, makes the operation in these cases a very severe and clumsy one, and the recovery long delayed.

3d. The action of the plug is to dilate the openings 
and the canal, instead of contracting them, and thus they are rendered more ready to admit hernial protrusions than before. The principle of plugging up in this manner a dilatable opening with the idea of closing it, is surely a false one. In the cases I have examined after Wurzer's operation has been performed, the external ring and the canal have been remarkably patulous.

4th. The elastic reaction of the skin, and the weight of the testis and scrotum, are enabled by this patulous condition of the external ring, to drag down the invaginated tissues, by exerting their force upon the adhesions at the upper part of the canal, and thus aiding in the dilatation of the posterior fold. The hollow cone of skin is usually totally obliterated by this process in a greater or less interval of time after the operation.

From what I have seen in the treatment of these cases, I believe that much of the mass of inflammatory thickening and exudation obtained by artificial means, is temporary only; the parts sooner or later assuming their normal flexibility and mobility. But if by means of the adhesive process a close approximation of the normal and permanent transplanted structures is obtained, these afford in themselves the best obstacle to the descent of the bowel. The absorption of the mass of exudation is, I am convinced, accelerated by the constant wearing of a truss soon after the operation.

5th. In many cases with narrow canals, failure is owing to the impossibility of invaginating the skin as far up as the internal opening, a mere indentation only being accomplished, leaving at the top of the canal a portion of sac ready for the formation of a fresh hernia. In some cases I have operated on, I have found the sac much narrowed at the external ring, and widening considerably towards the inner opening, so as to be funnel shaped. These obviously. would not admit a sufficiently large plug to act on the internal opening, except by a preliminary process of dilatation of the external ring, which seems to be a painful process, and one objectionable in principle. The dilating 
plug used by Mr. Davies, of Birmingham, is intended to meet these cases, but does so, I have no doubt, at the expense of much pain to the patient and sloughing of the skin.

Now, in order to avoid these sources of failure in operating for the radical cure of hernia, it seemed to me better to proceed upon a principle directly opposite to that of dilatation employed in Wurzer's, namely, that of drawing together and compressing by ligature the abdominal opening and the inguinal canal, to cause to cohere closely together, by adhesion in the track of the encircling ligature, the structures which form their sides and boundaries, and to agglomerate a solid mass, not of new and adventitious tissue, but of the more permanent tendinous and fascial structures. And to accomplish this more effectually, both by obtaining a higher point within the canal, and a more distinct perception of the anatomy of the parts operated upon, and by lessening as much as possible, and at the same time rendering as effective and permanent as possible, the interposed and transplanted tissue, it seemed better to give the operation a subcutaneous character. These two principles combined in the use of a subcutaneous ligature are, so far as I am aware, new in their application to the radical cure of hernia, and, indeed, I am not aware that they have been employed in this combination by other operators in any other surgical proceeding. I relied the more on their truth, in that $I$ had been successful on many previous occasions, in the treatment of subcutaneous nævi on the face and other exposed situations, without producing a loss of skin or much scar, by the application of a "subcutaneous clovehitch," which was followed by a remarkable contraction and consolidation of the subcutaneous structures. One of these cases was published in the 'Medical Times and Gazette' some months ago.

Description of the operation. - The pubis and scrotum of the affected side being shaved, the patient should be laid upon his back with the shoulders raised. The surgeon will find it most convenient to stand on the side to be operated on. The hernia must then be reduced, and held up by an 
assistant pressing with his fingers over the internal ring. With a small, narrow-bladed tenotomy knife, an incision is made to the depth of the skin only, of about three quarters of an inch in length, in the scrotum, over the centre of the lower part of the heruial tumour, at least two inches below the spine of the pubes. (Diagram IV, fig. 5.) Then the knife, being insinuated flatways between the skin and fascia, is made to separate the former from the latter all round the edges of the incision, over an area of at least two inches in vertical, by one and a half inch in transverse, diameter. This, from the loose attachment of the skin at this part, is easily done. The knees of the patient should next be drawn up towards the abdomen, and held together, so as to relax the structures connected with Poupart's ligament. Then the forefinger of the operator is passed through the opening in the skin, and made to invaginate the detached fascia through the external ring into the inguinal canal. This will be most effectually done by using the right hand for the right side, and vice versal. The operator should endeavour to commence the invagination of the fascia from as low a point as the external incision will admit, so as to push the finger as much as possible between the sac of the hernia and the spermatic cord, which should, at the same time, be put on the stretch by traction upon the testis with the disengaged hand. (Pl. V, fig. 1.) The invaginating finger should be made to reach the internal opening of the hernia.

The position of the spermatic cord, lying above and behind Poupart's ligament, can then be distinctly made out. When the point of the finger has reached the internal opening, it will be placed behind the lower border of the internal oblique muscle, the position of which will be made more evident to the operator by hooking it forward upon the point of the finger. (Pl. V, fig. 2.) He will then be sensible of the edge of the conjoined tendon (into which the muscle is inserted internally) at the outer side of his finger (in reference to his own hand) passing downwards behind the canal, to be inserted with Gimbernat's ligament 
into the pectineal line of the pubis. Next, a stout, bluntpointed needle mounted on a strong handle, with a wellmarked curve (as seen in the figure), is to be passed along the outer border of the invaginating finger, and pushed through the conjoined tendon and internal pillar of the external ring, till the point is seen to raise the skin on the surface of the finger. In this manœuvre the point of the needle must be carefully preceded by that of the finger. The skin is then to be drawn inwards and a little upwards, as much as its attachments will allow it to slide, and the point of the needle pushed through. A strong, smooth, hempen thread (well waxed, and then soaped) is then passed through the needle's eye, and the latter withdrawn, leaving one end of the thread in the puncture. The invaginating finger is then passed behind Poupart's ligament, between it and the spermatic cord, as far from the edge of the external pillar of the external ring, and as near to the internal ring, as possible. The ligament may then be raised forwards towards the surface upon the finger. (Pl. V, fig. 3.) The needle carrying the end of the ligature, which it has before withdrawn, must then be passed along the internal border of the invaginating finger to the deep surface of Poupart's ligament, about its centre (midway between the iliac and pubic spines), and pushed carefully through towards the skin. When its point begins to raise the skin, the latter is to be again slid outwards and a little downwards, till the point of the needle appears at the puncture before made: through this the instrument is to be a second time pushed, the loop of the thread seized, and the needle again withdrawn, leaving a loop in the puncture, and carrying the free end of the thread. The invaginating finger is then to be again placed on the inner side of the spermatic cord, which is to be pushed outwards by it. The point of the finger is then to be pressed against the centre of the posterior wall, so as to push backward the conjoined tendon, \&c. (Pl. V, fig. 4.) The thick, tendinous structure, formed by the triangular ligament and conjoined tendon, will then be felt covering the edge of the rectus muscle on the inner side of the 
finger, raised by the muscle considerably in relief. Into this the needle is to be next thrust, so as to take up a portion of it about half an inch above the pubic spine, close to its insertion into the pectineal line, and external to the border of the rectus muscle, which affords a very plain guide, and protects the peritoneum from injury.

By sliding the original puncture in the skin downwards to its utmost extent, the needle can easily be passed a third time through it. The needle is then freed from the ligature and withdrawn. We have then, passing through the puncture of the skin in the groin, the two ends of the ligature and a central loop. The upper of the ends passes through the upper part of the conjoined tendon, where the fibres of the oblique muscle begin to be inserted on it; the lower, through the triangular ligament and conjoined tendon, close to the insertion of Gimbernat's and Poupart's ligaments into the pubis; and both through the aponeurosis of the external oblique forming the inner pillar of the ring. The central loop passes through the centre of Poupart's ligament and the lower fibres of the internal oblique muscle arising from it. Thus, we leave in the canal two threads, passing across the invaginated fascia, and, in large and old cases, across the sac, immediately above and in front of and investing the spermatic cord, and connecting the inferior and external boundary of the canal, or Poupart's ligament, with the superior, internal, and posterior boundaries, formed by the fascia transversalis, conjoined tendon, and triangular ligament. (Pl. IV, fig. 2.) A pad of boxwood, or, what is still better, of glass or porcelain (c), about two inches long and one inch wide, flattened so as to be oval in section, and with the ends rounded off, is next applied obliquely upon the skin, along the course of the inguinal canal, with the centre opposite the puncture. The two free ends of the ligature are drawn to the outer side, and the loop to the inner side of the pad, so as to cross beneath it. One end of the thread is then passed through the loop and tied in a loop-knot to the other. The advantages of the double ligature are-1st, that of additional security 
against immediate protrusion; 2dly, that of producing action in the whole extent of the canal by obtaining two tracks and a broad intervening surface of adhesive effusion across the sac and fascia, one close to the internal ring, and the other below, near the external; and 3dly, the more effectual occlusion of the external ring, upon which the lower ligature directly acts. By keeping the two ligatures upon one thread, and making one knot suffice, we obtain a more equable adjustment of the pressure when the swelling supervenes. By tightening the string before the pad is placed, the surgeon may obtain direct evidence of the effect of the ligatures in closing the canal, on placing his finger therein. If the ligatures be properly placed, the canal will, even though a very wide one, be found completely occluded. The posterior wall should be felt drawn forward to meet the anterior. If this be not distinctly perceived, I consider the threads are not properly placed. I may here remark that the success of the operation mainly depends upon the effective closing of the canal by this drawing together of its anterior and posterior walls, and I have no doubt that many fuilures in this point will happen to the surgeon till he becomes accustomed to the manipulation of the parts. After the ligature is tied firmly over the compress, the lower end of the latter should reach nearly to the pubic crest, up to which point the wound in the scrotum will be tucked. Pledgets of lint may then be placed on each side of the compress, and a stout linen pad over all, secured by a spica bandage. The patient should be carried to bed, his shoulders raised, and a long bolster under his knees. If he has a bad night, and much pain in the groin, the removal of the linen pad and bandage will much relieve him. As their value ceases after the first twenty-four hours, and they may be injurious in retaining any discharge that may form, I have lately made it a rule to remove them the next day. Pressure is beneficial only so long as there is a chance of the deeper-seated parts becoming adherent by the first intention. In some cases, the discharge is very trifling, the lower opening 
healing at once. In most the discharge is greater, being at first serous and reddish, but soon becoming thick and purulent. In large cases, where the sac is punctured, the serous discharge is sometimes abundant, and evidently proceeds from the interior of the sac. In one of my cases the sac below the ligatures sloughed; and the discharges and sloughs escaped freely by the lower opening. The ligatures caused effectual adhesion of the neck of the sac, and thus prevented extension of mischief to the peritoneum. This patient made an excellent cure, and was, at the same time, relieved of a varicocele which coexisted with the hernia. In no case should the lower opening be stitched up, as it is very useful if there be much discharge. The introduction of a drainage tube $I$ have, in one case, found beneficial. In small cases, or where sufficient action seems to be quickly set up, I remove the compress at the end of the third or fourth day; in more sluggish cases at the sixth or seventh day; leaving, however, the threads in the puncture to act as a seton in promoting the escape of the discharges, and at the same time prolonging the effusive process in their track, and in the canal and sac, for as long as may be deemed necessary. The amount of solid effusion may be estimated by the swelling and hardness felt at the external ring, in the canal, and in the track of the ligatures. If the inflammation run high, and the discharge be copious, a poultice or water-dressing may be applied after fomentations. In the latter stage of the cure, a sulphate of zinc lotion may be injected with benefit.

The results intended to be obtained by the operation just described may be briefly recapitulated as follows. I may with advantage place them in the order of the importance which I attach to them severally.

1st. The posterior and superior boundaries of the dilated canal are drawn forwards and downwards towards Poupart's ligament, and become united by inflammatory effusion, in the area of pressure exercised by the ligatures, to the anterior and inferior boundaries. By the use of the two ligatures, this takes place from the internal opening above,

XIIII. 
to the external ring below. The effect of this adhesion will be seen in Plate IV, by comparing figs. 1 and 2 with 3 and 4 , to make the posterior wall act like the limb of a valve $(v)$, excluding the bowel by closing against the anterior wall $(a)$. This action is aided by the contraction of the cicatrized tissues, and increased by the subsequent downward traction of the testis and scrotum. In this way we have an assurance, that the older the cure and the more the pressure, the greater the mechanical resistance and security against the return of the protrusion. The spermatic cord is closely embraced by the contracting tissues in the groove behind Poupart's ligament, which protects it from undue pressure.

2dly. The consolidation and contraction of the tendinous boundaries of the canal, in the track of the ligatures, render them more capable of resisting pressure from within, and so increase the strength of the parts afterwards. The effects of these cords of adhesion will he best understood by reference to Plate IV, fig. 3. It will be seen that the cicatrix in the skin of the groin, as seen in a vertical section at $a$, will be drawn backwards by the contraction of the circular cord of effusion, towards the internal parts and peritoneum at $v$ and $p$, compressing more and more closely the opposite walls at the upper and lower extremities of the inguinal canal. Before fully estimating the importance of this result, it must be noticed that the primary dilatation of the canal in the formation of oblique inguinal hernia, is chiefly produced by the yielding of the superior and posterior walls at the upper part of the canal; the aponeurotic tendon of the external oblique at first resisting the anterior protrusion, and then directing it downwards and inwards in the course of the canal towards the external ring. This produces, in many hernix, and indeed in most which are still bubonoceles, an infundibular form, with the apex at the external ring. In most patients who have a tendency to hernial protrusion, a bulging is apparent at the internal ring, from a deficiency in the development of the lower fibres of the internal oblique, which cover that opening in front. In muscular subjects, 
this is rendered much more prominent during the contractions of the recti muscles, which, by virtue of their compressing action upon the bowels, draw backwards with them their sheath, formed by the conjoined tendon above the point of splitting of the internal oblique portion of it. In these subjects there exists a disproportion between the muscular contracting force of the recti and the tendinous and muscular resisting forces at the internal ring; and the upper part of the canal is opened, as it were, by the backward traction of the recti muscles upon the conjoined tendon, as well as by the yielding of the anterior wall.

This action, associated with deficient development of the lower part of the internal oblique, appears to me to be the main cause of inguinal herniæ in muscular subjects. It will be seen that the two internal ligature-tracks of adhesion directly counteract the effects of this action, by drawing the cicatrix of the skin and anterior wall of the canal backward with the conjoined tendon, as may be seen upon the patients cured by this operation, by a depression of the cicatrix during the action of the recti muscles. It will also be seen that Poupart's ligament is thus made to act as a "point d'appui " of the resisting forces. It seems certain that no operation, which leaves the upper part of the canal unaltered, can produce a permanent cure of oblique inguinal hernia. The opening or sac which is thereby left affords an admission to a wedge of howel or omentum, and being acted upon by the whole power of the abdominal muscles, speedily enlarges at the expense of the loosely attached and abundant peritoneum opposite the part. The return of the hernia in such circumstances is only a question of time.

3dly. The invaginated and transplanted fascia adheres on its opposing raw surfaces by means of a cord-like connective tissue, which is formed in front of and is adherent to the spermatic cord : by similar fibrous tissue formed in the tracks of the ligatures the fascia is further held firmly within the canal, and connected with the skin at the upper cicatrix, as well as with the anterior and posterior walls. (Plate IV, fig. 3.) To obtain this result, as well as to gain a more distinct per. 
ception of the parts within the canal, is an object, I think, clearly worth an incision of a half or quarter of an inch long in the skin of the scrotum, and a separation of the fascia. Nor can I agree with the principle of invaginating the skin, and holding it in the canal by ligatures attempted to be passed through the pillars of the external ring. By passing the skin through the external ring, the borders of the ring are separated, and prevented from adhering together. Again, without the incision it is quite impossible to get a hold with the needle upon the conjoined tendon; and not only are the pillars of the external ring insecurely fixed, but the cord and other important structures are much endangered by the instrument.

4thly. The fresh adhesions of the scrotum to the pillars of the external ring, while the latter are held together by the ligature, contribute much to close it, to bind it to the cord, and to hold up the transplanted fascia in its new position in the canal. The closure or obliteration of the external ring by the lateral traction of the lower ligature drawing together the pillars, may be remarked as one of the original features of the operation, and as contributing much to its success. In small cases of direct inguinal hernia it may be even sufficient to produce a cure, if care be taken to secure a hold with the inner end of the ligature upon the triangular fascia (or ligament) forming the posterior wall at this point and covering the edge of the rectus muscle.

Lastly. In those cases where the sac is large, has become adherent to the cord, and cannot be pushed back into the peritoneal cavity, the punctures through the sac, and the passage of the ligature-threads across it in front of the cord, as well as the grip of the ligature around it, produce adhesion of its surfaces to each other and to the spermatic cord, over the whole extent of the invagination, and not merely in its anterior fold. At the same time it becomes firmly united in one mass with the walls of the canal which are tied up with it. In recent cases, where the protrusion was but small, or a "bubonocele," I believe the patient to have been cured by obtaining the first four results, without 
puncturing the sac at all, but only by pushing it up before the invaginating finger, and working with the needle altogether outside it.

In noticing the objections that have been made to the operation, since it was first brought before public notice in May, 1858, I must premise that they have mainly, if not entirely, been founded upon prima facie and theoretical grounds, or upon the results of operations said to be according to my method, but for the execution of which the plan is in no wise responsible. First, as regards the danger of the plan. It has been urged that the peritoneum, the spermatic cord, the epigastric vessels, and even the iliac vessels and bowels, are endangered by the passage of the needle. But it will be seen, by an inspection of Plate V, that if the operation be properly and carefully done, the invaginating finger of the operator is placed so as to fill up the internal opening and keep out the bowel, and also to intervene between the spermatic cord, and the fascia transversalis covering the epigastric vessels and peritoneum, on the one hand, and the end of the needle at the point of puncture, on the other.

The fascia transversalis is a structure of much importance in this operation, on account of its thickness and density, and the way in which it covers and protects the iliac vessels and their branches, the circumflex iliac and epigastric. It can readily be felt, by its resistance to the finger pressing back towards the peritoneum. Of course, some familiarity with the parts is essential to the hand of the surgeon before doing this operation on the living subject. Before passing the needle through Poupart's ligament, that structure should be raised forward upon the finger and lifted, as it were, from the femoral vessels below and behind it, which may, at the same time, be pressed backward and outward by the thumb of the invaginating hand, placed on the skin below. (See Plate V, fig. 3.)

The needle used in this operation is much curved. The concavity of the curve should be kept carefully directed towards the surface, to which the point must necessarily 
tend if this precaution be adhered to, the needle kept steady, and carefully protected with the point of the invaginating finger. The point of the instrument should taper abruptly, and be well rounded on the convexity; and on no account should it have a cutting edge. It should be adapted rather for splitting the tissues, than for cutting its way through; and should therefore not be too sharp. If this be not attended to, the tendinous fibres of Poupart's ligament may be cut as by a tenotomy knife, instead of punctured; and so the hold of the ligature upon it be destroyed.

The best answer to any objection as to the danger of peritonitis from interfering with parts so near to the peritoneum, is to be drawn from the total results of my cases, fifteen in number, and all inguinal herniæ, in addition to the great number of cases in which Wurzer's plan, which interferes rather more with the peritoneum than mine, has been followed without bad results. The severity of an operation is best judged of by the duration of the after-treatment. In only one case was the patient detained in bed for more than a month. This was a case in which copious suppuration and burrowing of matter between the muscles of the abdomen supervened about the sixth day, with tympanitis, possibly associated with some parietal peritonitis. Of the rest of the cases, one was up on the ninth day, one on the eleventh, two on the twelfth, two on the fourteenth, four on the eighteenth; one three weeks after, another one month. All have done well as regards safety to life. In all, except two or three, the general symptoms were so slight as to excite little attention. These results can hardly be said to support the objection of severity or danger. Nor can the slight incision in the scrotum, which favours the downward escape of the discharge, be considered to make this operation more severe, or more likely to be followed by mischief, than Wurzer's or Gerdy's, in which the instrument also punctures the scrotum. I ought to mention that as little disturbance of the tissues as possible is desirable in the operation. Much roughness 
increases the chances of bad results, of abscesses and inordinate inflammatory action.

Of the efficacy of the operation, the best proof lies also in the results of the cases; and, as there is no proof like ocular proof, I have in attendance to-night, for the inspection of the Fellows, six cases in which a cure has been effected. One is the first case on which I operated, one year and eleven months ago. Another is the patient in whom the symptoms were most severe after the operation; the cure in whom has been so perfect, that from the time of the operation he has worn no truss for four months. I have also present the patient in whom the cure was one of the most speedy, and the symptoms the most slight; he having been out of bed on the twelfth day after the operation. In this patient the permanence of the cure has been tried eight months, and most severely tested, first by a bronchitis, which came on during the progress of the cure, and next by his having been employed without truss in the laborious work of harvesting and forking hay last autumn. Another of the cases present has been cured of a congenital hernia, which has been thought most difficult to cure. This patient was operated on last June (eight months ago), and has worn no truss since the operation, nor before, although he has been subject to heavy labour (piling shells) at Woolwich. A fourth case is here for inspection, in which no truss whatever has been worn before or since the operation, which was done eight months ago. This is also a very complete cure, the only trace of the deformity being a slight bulging opposite the internal ring (present also on the other side), from tenuity of the abdominal wall. The cough impulse is evidently arrested above the upper cicatrix.

The most likely cause of failure in this operation consists in the operator placing his ligatures too low, and closing the external ring only, whereby he fails to obtain a hold on the posterior wall of the canal. This, though effectual in closing the external opening, merely converts the hernia into a "bubonocele;" and, since the attachment of the 
oblique muscles to each other is not very intimate, the hernia may reach a considerable size, and even become strangulated by protruding between them, and without passing into the scrotum at all. It is then only a question of time as to its descent into the scrotum; the power of the abdominal muscles being so great, that when once a sac, however small, admits the fluid pressure of a piece of intestine, the complete dilatation of the rings and canal must take place sooner or later, unless a truss is worn. The only efficient closure is the valve-like approximation of the sides of the canal. One other cause of failure consists in the stitches not being placed far enough on the posterior wall, but so as to permit the intestine to slip down behind them. This I have seen happen in more than one instance. The threads should so be placed, that the opening is completely stitched up, so to speak.

In all the operations that have been hitherto proposed for the cure of hernia, it has been thought necessary to cause a truss to be worn for some months after the operation. In my first cases I followed the usual plan; but, thinking that the pressure of the truss rather promoted the absorption of the newly formed tissues, I have, in the last five or six cases, dispensed nearly altogether with its use. In four of the cases that are present this evening no truss has been worn. And though it was partly with a view of more thoroughly testing the efficacy of the cure, as well as of giving more tenacity to the adhesions, that $I$, at first, left off the constant use of the truss, I am at present disposed, in favorable cases, not to recommend the wearing of a truss after the operation. If, however, the abdominal parietes at the internal ring be weak and bulging after the operation, as is often the case, even when no hernia has shown itself, the use of a weak truss, with a flat pad, during violent exercise or hard work, is advisable. Trusses with strong springs are, I believe, most injurious ; if worn constantly, they favour very much the absorption of the effusion within the canal, and have, in my experience, given rise to varicocele. The occasional and intermittent use of a weak 
instrument is, on the contrary, beneficial, by exciting contraction in the canal. In two cases, after a sufficient length of time had elapsed to test the reality of the cure, I recommended the occasional use of a weak truss with this view. The usual effect of a truss worn continuously after an operation for the radical cure is to mask its failure. Perhaps the operation has diminished somewhat the internal opening. The accustomed knuckle of bowel does not find its way so readily into the canal. The hernia does not descend until the removal of the truss permits the effect of the abdominal muscles upon it. For this reason I think it desirable to test the reality of the cure before the truss is put on; and only in cases with weak, bulging groins should the truss be habitually worn.

In only one of the cases has the omission of the truss clearly given rise to a relapse; and this was entirely from the folly of the patient, a sailor boy, who very imprudently, and from over-confidence, attempted to lift a hundredweight of coals, three weeks or a month after the operation, and without a truss. The consequence, as might have been anticipated, was a rupture of all the adhesions and a reproduction of the hernia. It ought to be said, however, that in this case one ligature only had been used, the patient was only in bed nime days, and the cause of the return of the hernia was a greater effort than had been sufficient for the production of the original hernia. 


\section{EXPLANATION OF PLATE IV.}

Fig. 1. Diagrammatic section, showing the posterior fold left after invagination of the scrotum and sac in Gerdy's and Wurzer's methods of operating.

$a$. The anterior point of adhesion.

$v$. The posterior wall of the hernial canal.

s. The posterior fold of the sac or space in the canal into which the intestine re-descends.

Fig. 2. A similar section, after the author's method, showing the course, position, and effect of the ligature and compress upon the invaginated fascia and sac and walls of the canal.

c. The compress, with the loop and ends of the ligature previous to tying.

$v$ and $p$. The upper and lower perforations of the posterior wall (conjoined tendon).

Fig. 3. Ditto, showing the adhesions of the above-named structures after the operation, the consolidation of the anterior and posterior walls, and the circular contraction in the track of the ligatures.

Fig. 4 illustrates the valve-like action of the posterior wall in preventing the descent of the intestine.

Fig. 5 illustrates the first step in the author's method of operating, viz., the puncture in the scrotum and the separation of the fascia from the skin by the subcutaneous knife. 

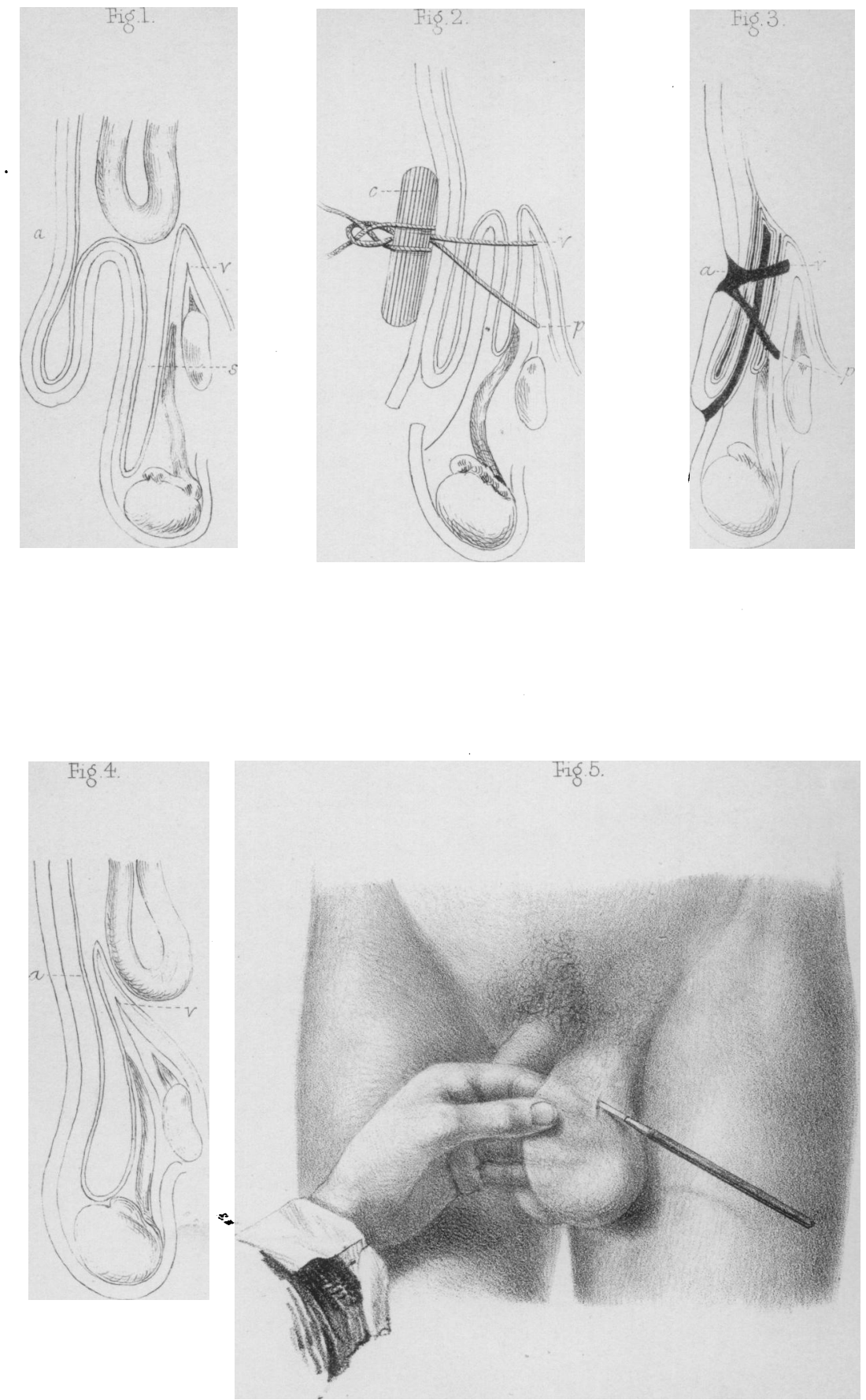

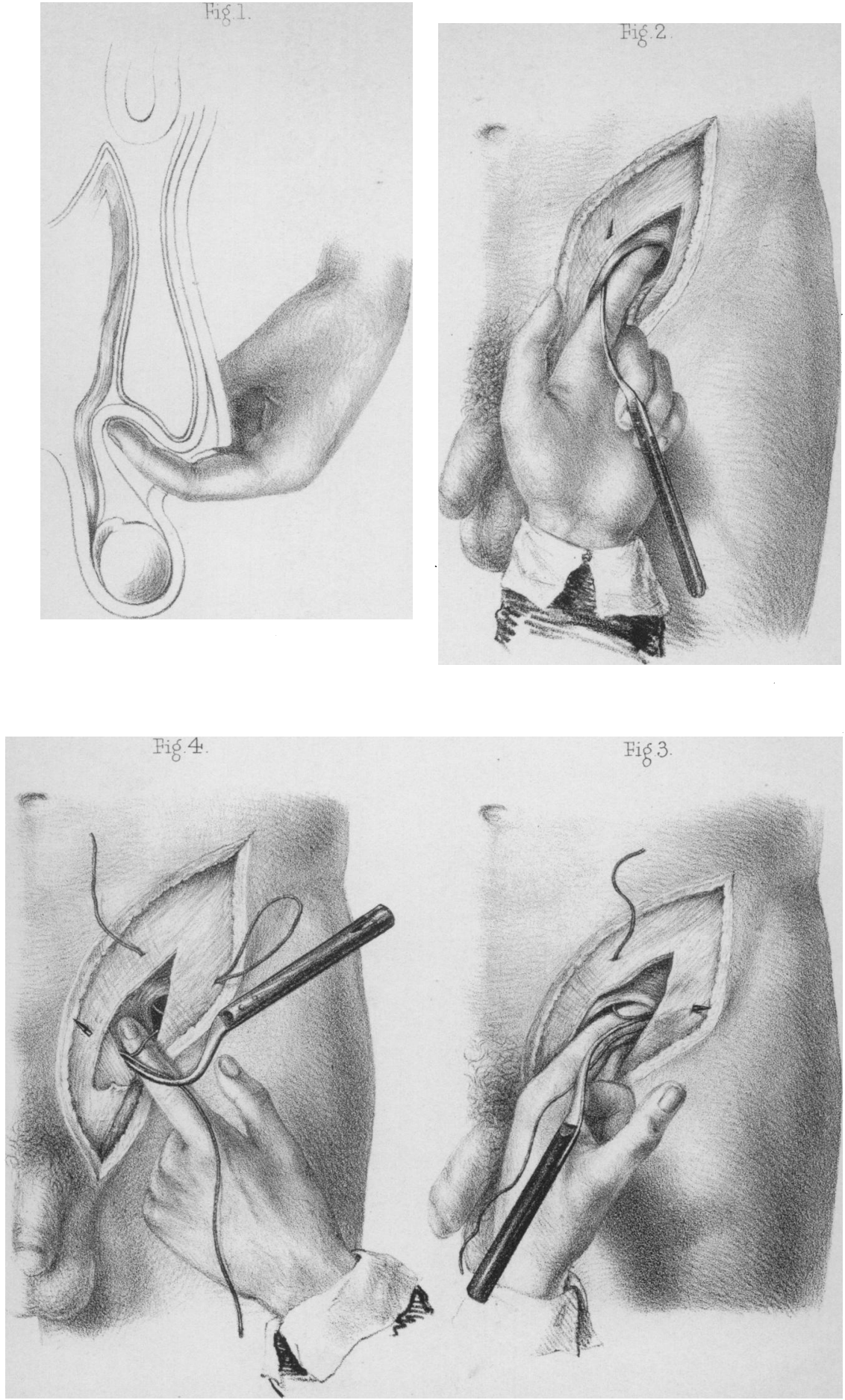


\section{EXPLANATION OF PLATE V.}

Fig. 1. Sectional drawing, illustrating the second step in the author's operation, viz., the passage of the forefinger through the skin of the scrotum, and the invagination of the fascia and sac.

Fig. 2 illustrates the third step in the operation, as exposed by a dissection of the integuments outwards. The aponeurosis of the external oblique is slit up in the site of the external ring, to show the forefinger of the operator raising the lower border of the internal oblique muscle, and the needle passing through the upper part of the conjoined tendon.

Fig. 3 shows the next proceeding. The end of the ligature is left in the first puncture; the surgeon's finger raises the external pillar directly above Poupart's ligament, and guards the point of the needle as it passes through, protecting the spermatic cord and deep vessels.

Fig. 4 shows the application of the lower end of the ligature to the triangular fascia, conjoined tendon, and internal pillar close to the pubis. The finger of the operator is seen to draw outwards the spermatic cord, and to protect it from the needle. The loop of the ligature is left in the second puncture, and the lower end of it through the last. 